\title{
Spatio-Temporal Expression Profile of Mettl3/Mettl14 During Mouse Retina Development
}

\author{
Perfil de Expresión Espacio-Temporal de Mett13 / Mettl14 \\ Durante el Desarrollo de la Retina del Ratón
}

Qi Zhong'ㄹ Jingyi Guo ${ }^{1}$ \& Shuyi Chen ${ }^{1}$

ZHONG, Q.; GUO, J. \& CHEN, S. Spatio-temporal expression profile of Mettl3/Mettl14 during mouse retina development. Int. J. Morphol., 38(6):1668-1675, 2020.

SUMMARY: The Mettl3/Mett114 methyltransferase complex installs the most ubiquitous internal mRNA modificationN6-methyladenosine (m6A). The vertebrate retina development is a multi-step process that requires fine-tuning of multiple cellular events, but very little is known about the potential function of Mettl3 and Mettl14 in this process. In this study, we demonstrated the spatio-temporal expression of Mettl3 and Mettl14 during retina development in mouse by quantitative PCR and immunofluorescence staining. We found that these two components of methyltransferase complex could be detected from the beginning of retina development; and the expression of Mettl3 and Mettl14 were gradually restricted to inner nuclear layer (INL) and ganglion cell layer (GCL); Double labeling showed that Mettl3 and Mettl14 had similar expression patterns in mature retinal INL and GCL. Overall, our spatio-temporal expression data provided the foundation for future research on the function of m6A modification in the retina development.

KEY WORDS: m6A methyltransferase; Mettl3; Mettl14; Retinogenesis; Expression.

\section{INTRODUCTION}

The study of retinal development and retinopathy requires an understanding of various cellular mechanisms that regulate gene expression. Much work has focused on lineage-specific transcription networks that govern stem cell differentiation (Furlong \& Levine, 2018). There are more than 170 kinds of modifications in RNA, among which m6A is one of the most abundant and well-studied mRNA modifications. Among all transcriptomes encoded by mammalian cells, $20 \%$ to $40 \%$ contain N6methyladenosine (m6A) methylation modifications, and methylated mRNAs often contain multiple m6A modification sites in each transcript (Dominissini et al., 2012; Meyer et al., 2012). m6A and other RNA modifications also exist in long non-coding and microRNAs. Researchers around the world have identified many enzymes involved in m6A, including methylase, demethylase and methylation recognition enzymes which are called writer, eraser, and reader, respectively (Roundtree et al., 2017). The m6A modification is installed by a multicomponent methyltransferase complex. The core of the complex is a heterodimer of two proteins, Mettl3 and
Mettl14. Mettl3 and Mettl14 have methyltransferase homologous domains. However, the crystal structures only showed SAM and SAH densities in the catalytic cavity of Mettl3, indicating that Mettl3 is the sole catalytic subunit. In contrast, the catalytic cavity of Mettl14 adopted a closed conformation that could not accommodate SAM. Mettl3 is the unique catalytic subunit, and Mettl14 functions in structural stabilization and RNA substrate recognition (Wang et al., 2016; Zhou \& Pan, 2016).

It has been reported that $\mathrm{m} 6 \mathrm{~A}$ methylation regulated embryonic cortical neurogenesis (Yoon et al., 2017). In the mouse, the optic vesicle is formed through an invagination of the diencephalic neural tube at E9.5 (Fuhrmann, 2010). Retina is a thin sheet of neural tissue located on the inner surface of the posterior eye. The adult retina comprises six types of neurons [rod and cone photoreceptors, bipolar, amacrine, horizontal, and ganglion cells (GCs)] and one type of glial cell (the Müller glia), which are organized into three distinct cellular layers. These different cell types are generated from a pool of multipotent retinal progenitor

\footnotetext{
${ }^{1}$ State Key Laboratory of Ophthalmology, Zhongshan Ophthalmic Center, Sun Yat-sen University, Guangzhou 510060, China.
} 
cells (RPCs) in a sequence that is remarkably conserved across all vertebrates (Young, 1985; Agathocleous \& Harris, 2009). During embryogenesis in the mouse, GCs are generated first, followed by the production of cone photoreceptors, horizontal cells, and most of the amacrine neurons. Bipolar neurons, Müller glia, the remaining amacrine neurons, and most rod photoreceptors are generated postnatally. Up to now, nothing is known about the role of $\mathrm{m} 6 \mathrm{~A}$ modification during mammalian retina development in vivo. In this study, we focused on the expression of methylase complex of m6A in the mouse retina. Embryonic and postnatal mouse retina samples were collected to examine the spatio-temporal expression of Mettl3 and Mettl14 during retina development in mouse. Our data provides the foundation for future research on the function of m6A modification in the retina development.

\section{MATERIAL AND METHOD}

Animal Maintenance. This study was conducted in strict accordance with the recommendations of the Association for Research in Vision and Ophthalmology (ARVO) Statement guidelines. The research protocol with animal experimentation was approved by the Institutional Animal Care and Use Committee of Zhongshan Ophthalmic Center. To obtain time-matched embryos, wild-type C57BL/6J male and female mice were caged together in the afternoon and vaginal plugs were checked the next morning. Noon of the day when a plug was found was counted as embryonic day 0.5 (E0.5). The animals used in this experiment included 16 pregnant mice, 6 in each stage of postnatal day $0(\mathrm{P} 0)$, P3, P6, P9, P15 and adult mice.

Quantitative PCR. Tissues were collected in TRIZOL Reagent (Invitrogen; California, USA) and RNA was isolated according to manufacturer's protocol. After cDNA was synthesized using Superscript III First-Strand Synthesis SuperMix (Invitrogen), quantitative PCR was performed using individual gene-specific quantitative PCR (qPCR) primers and the SYBR green dye (04887352001, Roche; Basel, Switzerland). The data was normalized using Gapdh as the internal control gene. The $\Delta \Delta \mathrm{Ct}$ value was calculated by difference in normalized $\mathrm{Ct}$ value $(\Delta \mathrm{Ct})$ from different periods to the $\Delta \mathrm{Ct}$ from E11.5. The $\Delta \Delta \mathrm{Ct}$ value was transformed into $2^{-\Delta \Delta \mathrm{Ct}}$ value as the estimated gene expression fold change value. Primer sequences for qRTPCR analysis were listed in Table I.

Immunostaining. Mouse retinas were fixed on $4 \%$ paraformaldehyde (PFA) in phosphate buffer for $4 \mathrm{~h}$ at room temperature. After fixation, retinas were dehydrated in $30 \%$ sucrose solution until embryos sank, and embedded into OCT (SAKURA; Torrance, USA). The blocks were cut into slides using Leica Cryostat Microtome (Leica CM1950; Wetzlar, GER). Slides were incubated with primary antibodies at $4^{\circ} \mathrm{C}$ overnight, washed with PBST, and then incubated with secondary antibodies at room temperature for 2 hours. The slides were then washed, counterstained with 4', 6-diamidino-2phenylindole (DAPI) for 10 minutes, washed again, and then mounted with cover glass using VECTASHIELD mounting medium (H-1000, Vector Laboratories; Burlingame, USA). Images were obtained with Nikon C2 confocal microscope (Nikon Eclipse Ni-E; Tokyo, JPN). Primary antibodies used in the study: monoclonal rabbit anti-Mett13 antibody (ab195352, 1:500; Abcam; Cambridge, USA), polyclonal rabbit anti-Mettl14 antibody (HPA038002, 1:500; Sigma-Aldrich; Darmstadt, GER), monoclonal mouse anti-Brn-3a antibody (MAB1585,1:100; Millipore; Boston, USA), monoclonal goat anti-Calretinin antibody (AB1550,1:1000; Millipore), monoclonal mouse anti-Syntaxin (Hpc-1) antibody (S0664,1:500; Sigma-Aldrich) and polyclonal sheep antiChx10 antibody (AB9014, 1:100; Millipore). Secondary antibodies used in the study: Donkey anti-Mouse IgG (H+L) Highly Cross-Adsorbed Secondary Antibody, Alexa Fluor 488 from Thermo Fisher Scientific(Waltham, MA USA), catalog \# A-21202, RRID AB_141607; Donkey anti-Rabbit IgG (H+L) Highly Cross-Adsorbed Secondary Antibody, Alexa Fluor 568 from Thermo Fisher Scientific, catalog \# A10042, RRID AB_2534017; Donkey anti-Goat IgG $(\mathrm{H}+\mathrm{L})$ Cross-Adsorbed Secondary Antibody, Alexa Fluor 488 from Thermo Fisher Scientific, catalog \# A11055, RRID AB_2534102; Donkey anti-Sheep IgG $(\mathrm{H}+\mathrm{L})$ Cross-Adsorbed Secondary Antibody, Alexa Fluor 488 from Thermo Fisher Scientific, catalog \# A-11015, RRID AB_2534082. The concentration of all secondary antibodies was 1:2000.

Table I. Primers used for qRT-PCR.

\begin{tabular}{lll}
\hline S pecies & Gene & Primers \\
\hline mouse & Mettl3 & 5'-ATCCAGGCCCATAAGAAACAG-3' \\
& 3'-CTATCACTACGGAAGGTTGGG-5' \\
& Mettll4 & 5'-GGTGAGCAGCCGAAGCTGG-3' \\
& 3'-CACCAATGCTATCCGCACTC-5' \\
\hline
\end{tabular}




\section{RESULTS}

Temporal expression profile of Mettl3 and Mettl14 at transcriptional level. Retinogenesis in the mouse begins midway through the gestational period at embryonic day 10 (E10) and continues through the second postnatal week. First, retinal ganglion cells differentiate from retinal progenitors at E11 followed by horizontal cells, cone photoreceptors and amacrine cells. Bipolar cells, rod photoreceptors and Müller glial cells are born in the latter half of retinal development (Turner et al., 1990; Livesey \& Cepko, 2001). Mouse E11.5, E13.5, E15.5, E17.5, postnatal day 0 (P0), P3, P6, $\mathrm{P} 9, \mathrm{P} 15$ and adult (2 months) retinas were collected to perform quantitative PCR. We found that the expression of Mettl3 remained at a low level during early retina development. At P6, the expression of Mettl3 bursted and increased two-fold compared to that at P3 and continued to increase through adulthood (Fig. 1A). The expression pattern of Mettl14 was similar to that of Mettl3. However, the expression of Mettl14 was significantly higher than that of Mettl3 during early retina development at transcriptional level (Fig. 1B).

Mettl3 and Mettl14 immunostaining patterns in Developing Mouse Retina. We applied immunofluorescence staining to locate the expression of Mettl3 and Mettl14 at different stages of mouse retina development. Mettl3 and Mettl14, which are nucleoproteins, were shown to co-locate with DAPI staining. The expression of Mettl3 and Mettl14 were detected in the RPC early at E11.5 (Figs. 2A and 3A). Later at P6 these two proteins were concentrated on the INL and GCL (Figs. 2G and 3G) and it continued into the adult retina (Figs. $2 \mathrm{H}-\mathrm{J}$ and $3 \mathrm{H}-\mathrm{J}$ ).

\section{Localization of Mettl3 and Mettl14 in mouse INL and}

GCL. To identify the cell types express Mettl3 and Mettl14, we performed double labeling experiments using antibodies to Mettl3 and Mettl14 in combination with wellestablished markers for different retina cells in a mature retina (P30). Co-labelling with anti- Brn3a, a marker for retinal ganglion cells (Pan et al., 2005), showed that the Mettl3 positive cells in the GCL also expressed Brn3a (Fig. 4A). The Mettl3-positive cells that were not labeled by Brn3a in the GCL could be a displaced amacrine cell. Indeed, those Mettl3-positive cells co-localized with calretinin (Chandra et al., 2017) (Fig. 4B), which marks a subset of amacrine cells in the GCL. Then we showed that Mettl3 was expressed in the nucleus of the amacrine cells, surrounded by the amacrine cell membrane marker HPC1 (Inoue \& Akagawa, 1993) (Fig. 4C). To study the other positive signals of Mettl3 in the INL, we examined the distribution of Mettl3 in combination with $\mathrm{Chx} 10$, a wellestablished markers for bipolar cells (Rowan \& Cepko, 2004). We found that many Mettl3-positive cells colocalized with Chx10 (Fig. 4D). The expression patterns of Mettl 3 and Mettl14 closely resembled each other in the mature retina (Fig. 4A'-D'). These results demonstrated for the first time the precise cell types that co-localized with Mettl3 and Mettl14 in the adult mouse retina.

\section{Mett|3}

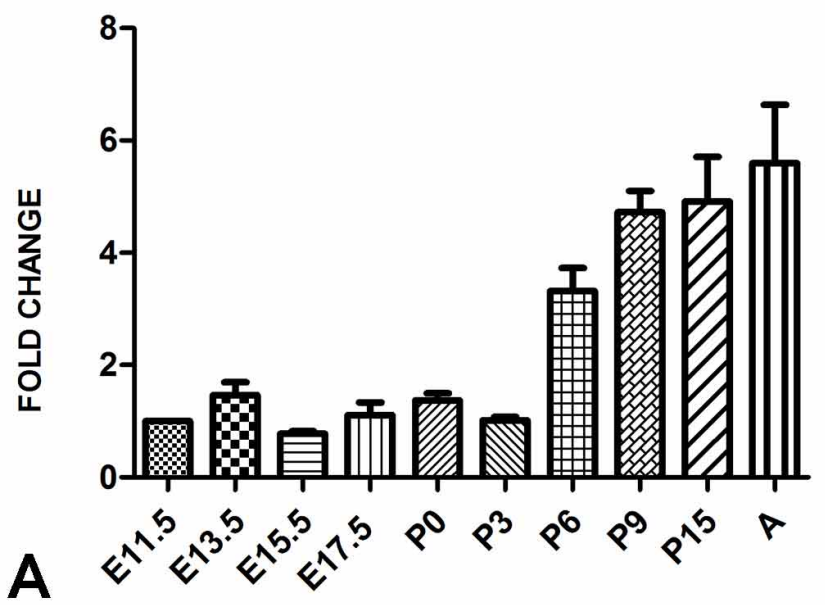

\section{Mettl14}

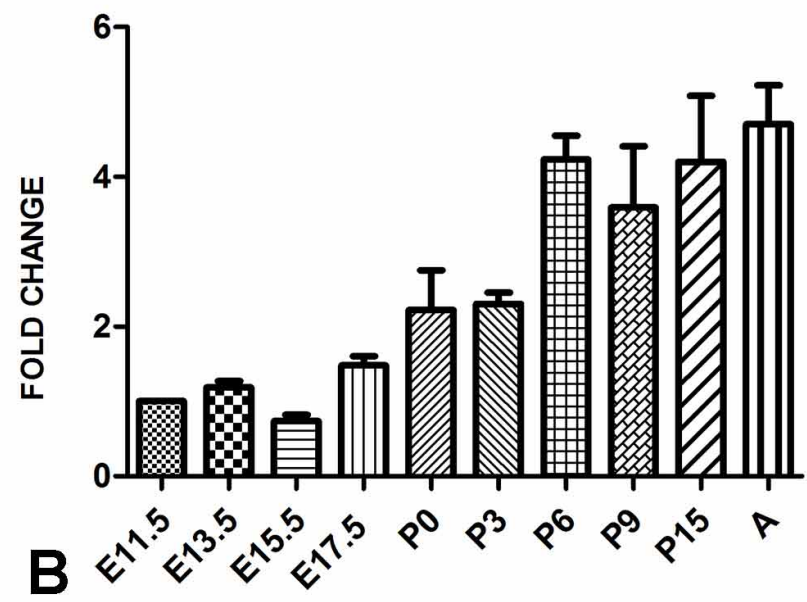

Fig. 1. Transcriptional level of m6A methyltransferase complex involved in mouse retinogenesis. (A, B) Quantitative Real Time PCR (qRT-PCR) of Mettl3 and Mettl14 in mouse retina cDNA samples at embryonic day 11.5 (E11.5), embryonic day 13.5 (E13.5), embryonic day 15.5 (E15.5), embryonic day 17.5 (E17.5) postnatal day 0 (P0), postnatal day 3 (P3), postnatal day 6 (P6), postnatal day 9 (P9), postnatal day 15 (P15) and Adulthood. Expression was normalized to GADPH (glyceraldehyde-3-phosphate dehydrogenase) gene expression, with all data points for E11.5 set to 1. 

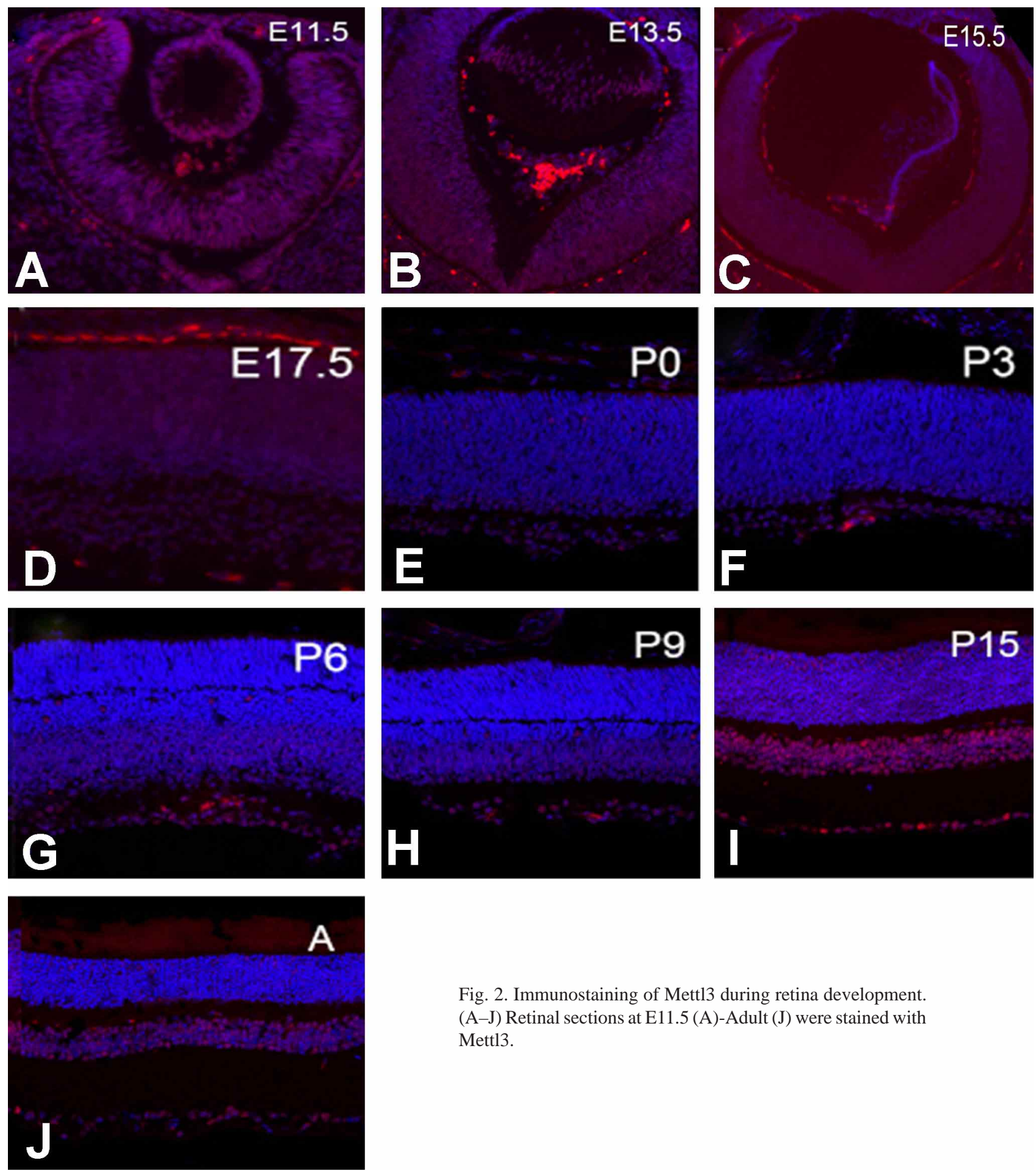

\section{DISCUSSION}

Normally, if the expression of a gene is found in the development of an organ or a tissue, the gene is generally considered to play a role in the development of that organ or tissue, the expression patterns of Mettl3 and Mettl14 during retina development have not been demonstrated yet, which are necessary for studying the role of $\mathrm{m} 6 \mathrm{~A}$ in retinogenesis, In the present study, we performed quantitative PCR and immunofluorescence labeling to show Mettl3 and Mettl14 expression patterns 

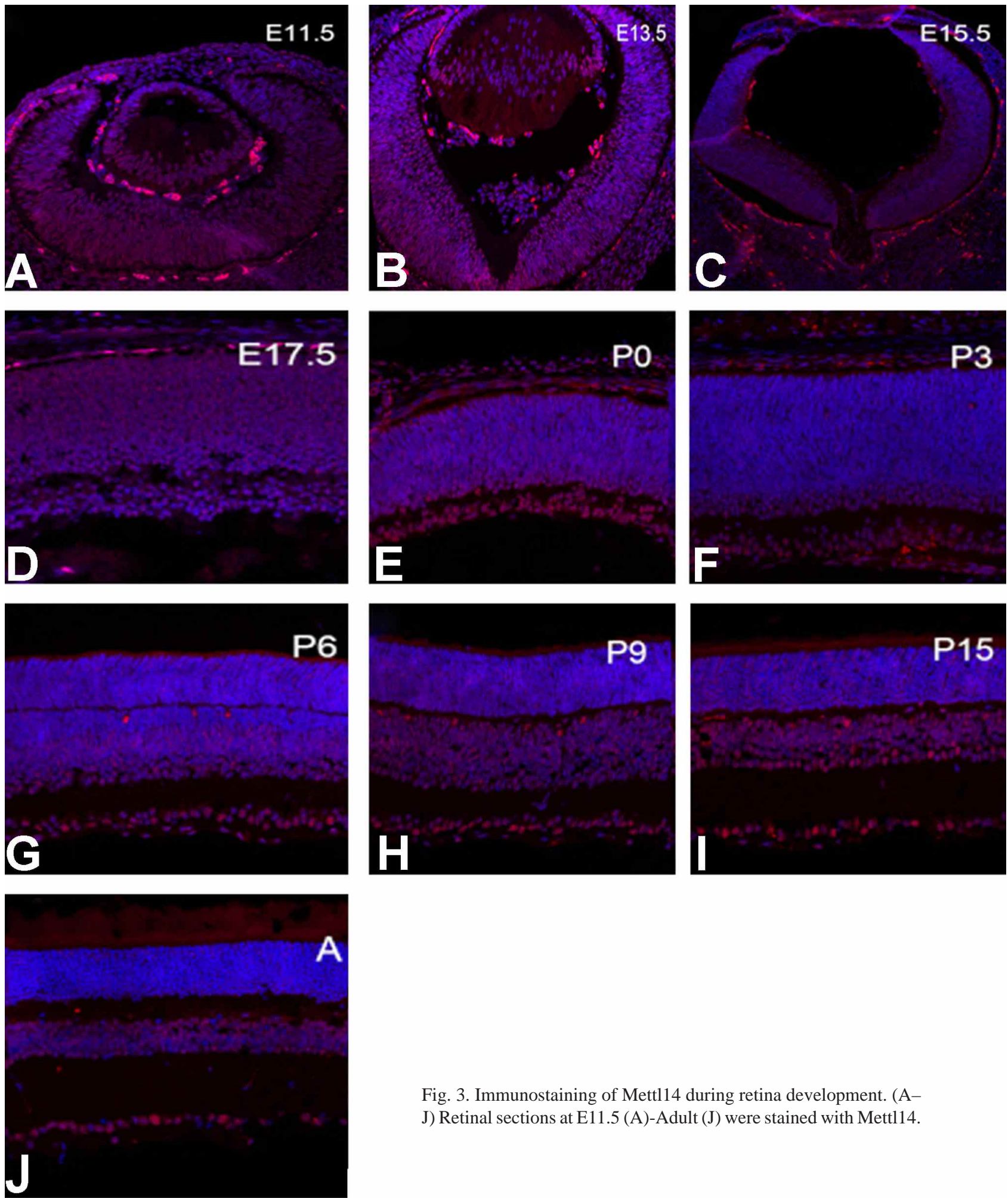

Fig. 3. Immunostaining of Mettl14 during retina development. (AJ) Retinal sections at E11.5 (A)-Adult (J) were stained with Mettl14.

during mouse retina development for the first time. We found that the expression of the two proteins runs through the entire retinal development process, we believe that m6A plays an important role in retinal development. In addition, the core component of m6A methyltransferase is a heterodimer containing two proteins Mettl3 and Mettl14. 


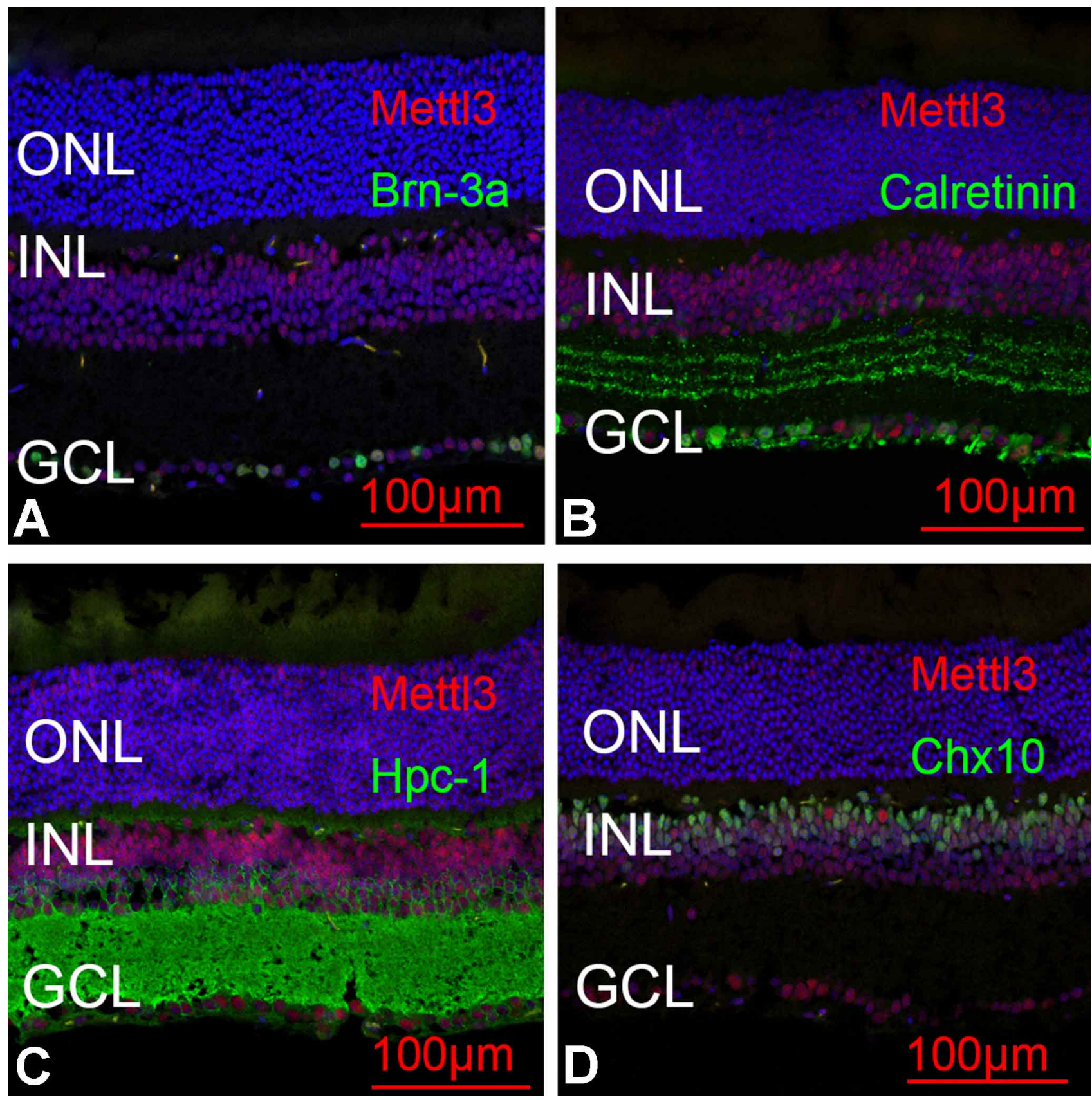

Fig. 4. Co-localize with Mettl3 and Mettl14 in the adult mouse retina. (A-D) Retinal sections at P30 were double stained with retinal markers (green) and Mettl3 (red). A: Mettl3 was expressed in RGCs and co-localized with strong Brn3a signals. B-C: Mettl3 was expressed in amacrine cells and co-localized with strong Calretinin and Hpc-1 signals. D: Mettl3 was expressed in bipolar cells and co-localized with strong Chx10 signals. ONL: outer nuclear layer; INL: inner nuclear layer; GCL: ganglion cell layer. Scale bars are $100 \mu \mathrm{m}$.

The highly consistent expression pattern of these two proteins echoes this fact. Finally, Immunofluorescence double labeling techniques showed that Mettl3 and Mettl14 were expressed in amacrine, bipolar and ganglion cells. In summary, Mettl3 and Mettl14 are broadly expressed and may play essential roles in the development and specification of mouse neuronal retina and glia cells. We believed that our work will provide a foundation for studying the functions of m6A during retina development. 


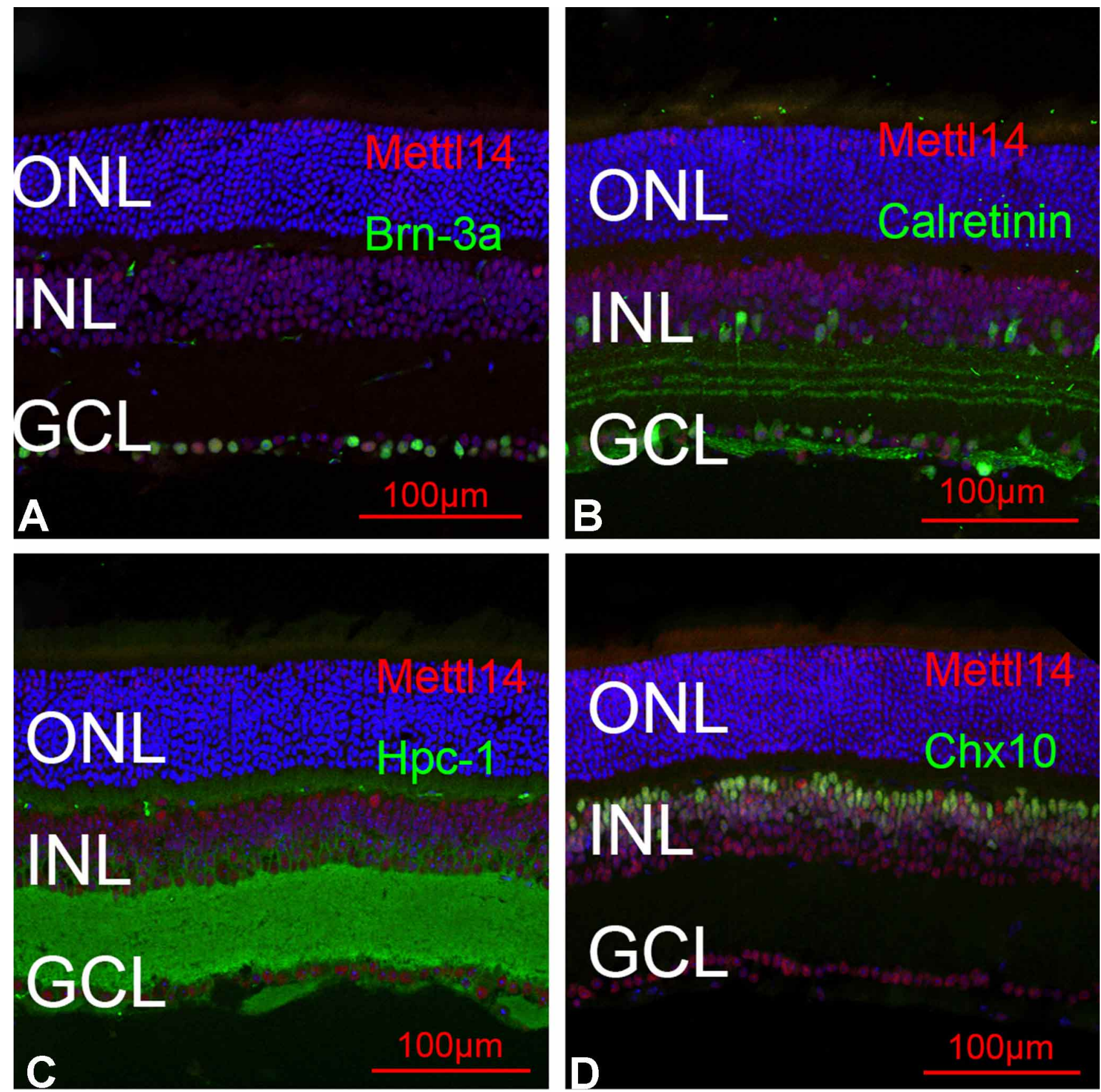

Fig. 5. Retinal sections at P30 were double stained with the same retinal markers (green) and Mettl14 (red) (A-D).

ACKNOWLEDGEMENTS. We acknowledge Ophthaimic Animal Laboratory of Zhongshan Ophthalmic Center for help.

ZHONG, Q.; GUO, J. \& CHEN, S. Perfil de expresión espaciotemporal de Mettl3 / Mettl14 durante el desarrollo de la retina del ratón. Int. J. Morphol., 38(6):1668-1675, 2020.

RESUMEN: El complejo Mettl3 / Mettl14 metiltransferasa establece la modificación interna más significativa de ARNm: N6metiladenosina (m6A). El desarrollo de la retina de los vertebrados es un proceso de varios pasos que requiere múltiples eventos celulares; existe muy poca información sobre la función potencial de Mettl3 y Mettl14 en este proceso. En este estudio, demostramos la expresión espacio-temporal de Mettl3 y Mettl14 durante el desarrollo de la retina en ratón mediante PCR cuantitativa y tinción de inmunofluorescencia. Descubrimos que estos dos componentes del complejo de metiltransferasa podían ser detectados desde el comienzo del desarrollo de la retina; la expresión de Mettl3 y Mettl14 se restringió gradualmente a la capa nuclear interna (INL) y la capa de células ganglionares (GCL); se observó que Mettl3 y 
Mettl14 tenían patrones de expresión similares en INL y GCL retinianos maduros. En general, nuestros datos de expresión espacio-temporal proporcionan información para futuras investigaciones sobre la función de la modificación de m6A en el desarrollo de la retina.

PALABRAS CLAVE: m6A metiltransferasa; Mettl3; Mettl14; Retinogénesis; Expresión.

\section{REFERENCES}

Agathocleous, M. \& Harris, W. A. From progenitors to differentiated cells in the vertebrate retina. Anпи. Rev. Cell Dev. Biol., 25:45-69, 2009.

Chandra, A. J.; Lee, S. C. S. \& Grünert, U. Thorny ganglion cells in marmoset retina: Morphological and neurochemical characterization with antibodies against calretinin. J. Comp. Neurol., 525(18):3962-3974, 2017.

Dominissini, D.; Moshitch-Moshkovitz, S.; Schwartz, S.; Salmon-Divon, M.; Ungar, L.; Osenberg, S.; Cesarkas, K.; Jacob-Hirsch, J.; Amariglio, N.; Kupiec, M.; et al. Topology of the human and mouse m6A RNA methylomes revealed by m6A-seq. Nature, 485(7397):201-6, 2012.

Fuhrmann, S. Eye morphogenesis and patterning of the optic vesicle. Curr. Top. Dev. Biol., 93:61-84, 2010.

Furlong, E. E. M. \& Levine, M. Developmental enhancers and chromosome topology. Science, 361(6409):1341-5, 2018.

Inoue, A. \& Akagawa, K. Neuron specific expression of a membrane protein, HPC-1: tissue distribution, and cellular and subcellular localization of immunoreactivity and mRNA. Brain Res. Mol. Brain Res., 19(1-2):1218, 1993.

Livesey, F. J. \& Cepko, C. L. Vertebrate neural cell-fate determination: lessons from the retina. Nat. Rev. Neurosci., 2(2):109-18, 2001.

Meyer, K. D.; Saletore, Y.; Zumbo, P.; Elemento, O.; Mason, C. E. \& Jaffrey, S. R. Comprehensive analysis of mRNA methylation reveals enrichment in 3' UTRs and near stop codons. Cell, 149(7):1635-46, 2012.

Pan, L.; Yang, Z.; Feng, L. \& Gan, L. Functional equivalence of Brn3 POUdomain transcription factors in mouse retinal neurogenesis. Development, 132(4):703-12, 2005.

Roundtree, I. A.; Evans, M. E.; Pan, T. \& He, C. Dynamic RNA modifications in gene expression regulation. Cell, 169(7):1187-200, 2017.

Rowan, S. \& Cepko, C. L. Genetic analysis of the homeodomain transcription factor $\mathrm{Chx} 10$ in the retina using a novel multifunctional BAC transgenic mouse reporter. Dev. Biol., 271(2):388-402, 2004.

Turner, D. L.; Snyder, E. Y. \& Cepko, C. L. Lineage-independent determination of cell type in the embryonic mouse retina. Neuron, 4(6):833-45, 1990.

Wang, P.; Doxtader, K. A. \& Nam, Y. Structural basis for cooperative function of Mettl3 and Mettl14 methyltransferases. Mol. Cell, 63(2):306-17, 2016.

Yoon, K. J.; Ringeling, F. R.; Vissers, C.; Jacob, F.; Pokrass, M.; JimenezCyrus, D.; Su, Y.; Kim, N. S.; Zhu, Y.; Zheng, L.; et al. Temporal control of mammalian cortical neurogenesis by $\mathrm{m} 6$ A methylation. Cell, 171(4):877-889.e17, 2017.

Young, R. W. Cell differentiation in the retina of the mouse. Anat. Rec., 212(2):199-205, 1985.

Zhou, K. I. \& Pan, T. Structures of the m6A methyltransferase complex: two subunits with distinct but coordinated roles. Mol. Cell, 63(2):183$5,2016$.

\author{
Corresponding author: \\ Shuyi Chen \\ State Key Laboratory of Ophthalmology \\ Zhongshan Ophthalmic Center \\ Sun Yat-sen University \\ Guangzhou 510060 \\ CHINA
}

Email: chenshy23@mail.sysu.edu.cn.

Received: 27-05-2020

Accepted: 07-08-2020 